\title{
Numerical Study of Sound Pressure Distribution in Volume Determination with Acoustic Method
}

\author{
Man-Hong HU*, Jian WANG, Chang-Qing CAI, Tao HUANG, Rui-Lin ZHONG \\ and Kai JIAO
}

\author{
Division of Mechanics and Acoustic, National Institute of Metrology, 100029, Beijing, China \\ *hmh@nim.ac.cn
}

Keywords: Sound pressure distribution, Volume measurement, Finite element method.

\begin{abstract}
Stable and uniform sound distribution is a key factor in high precise volume determination of solid material especially for mass standards. A numerical study is carried out to study the distribution and uniformity of sound pressure. The FEM simulation results show that the sound pressure distribution gradient of the small chamber is better than large chamber. With the FEM model in this paper, the maximum sound pressure level change is only 0.003 DB (RMS). And also for small chamber, the sound pressure signals are higher and more easily to be acquired for real measuring system.
\end{abstract}

\section{Introduction}

Volume determination of solid material is important in the air bouncy correction for mass metrology and industrial application. Acoustic method is a non-contact volume determination method based on gas compressibility laws. Acoustic method is based on gas compressibility laws. In state of gas is adiabatic, the air pressure, $\mathrm{P}$, has a constant relation with the volume of air, $\mathrm{V}$, as expressed in Equation1:

$$
P \times V^{\gamma}=\text { cons }
$$

When using a loud speak in a closed chamber, the sound pressure which is a physically pressure vibration based on air pressure, has the linear relation with the volume of closed chamber. Here, $\gamma$ is ratio of specific heats, which is 1.40 at atmospheric pressure and room temperature.

To determine the volume of a solid material such as mass standard, M. Ueki et al. firstly developed an acoustic measuring system to measure the volume of mass weights ranging from 100 $\mathrm{g}$ to $10 \mathrm{~kg}$ with a relative uncertainty of $1 \times 10^{-3}(k=2)$, and measure the volume of mass weights ranging from $1 \mathrm{~g}$ to $100 \mathrm{~g}$ with combined standard uncertainty is below $0.0021 \mathrm{~cm}^{3}$. [1-6] an acoustic volume measuring system has also been developed by National Institute of Metrology China (NIM) to extend the measurement range of mass weight up to $20 \mathrm{~kg}$. [7] The solid material's volume is determined by measuring to sound pressure in two closed chamber. For high precise volume measurements, the sound pressure in the closed chamber as an essential parameter needs to be stable and uniform.

To achieve higher volume determination accuracy, a numerical study is carried out to study the distribution and uniformity of sound pressure. And experiments are also carried out to evaluate the effects of numerical study, and the uncertainty of measuring results is discussed.

\section{Numerical Simulation Methods}

Figure 1 shows the schematic diagram of self-designed measuring apparatus made of aluminium alloy with two measuring chambers. The signal generator will generate the sine drive signal to the loud speaker located between the two closed chambers. The loud speaker will vibrate and compress the air in two chambers with same amplitude and reversed phase, and will generate two sound pressures in the two chambers. Two sound pressure sensors locate in the side wall of chamber, will 
acquire the sound pressure signal. Through the amplifier, the sensing signal will transfer to the control computer for calculation the sound pressure.

The acoustic volume measuring system has no complicated intake and exhaust system. The design principle is to get high accuracy and stability of sound pressure. The two measuring chambers have the structures of simplified cylindrical shape as showed in Figure 1. And the calculation of the sound pressure equations in the actual systems is difficult to obtain analytical solutions. Therefore, the finite element modelling (FEM) and simulation method is applied to the optimization analysis and design of the measuring stability and accuracy of the acoustic volume measuring system.

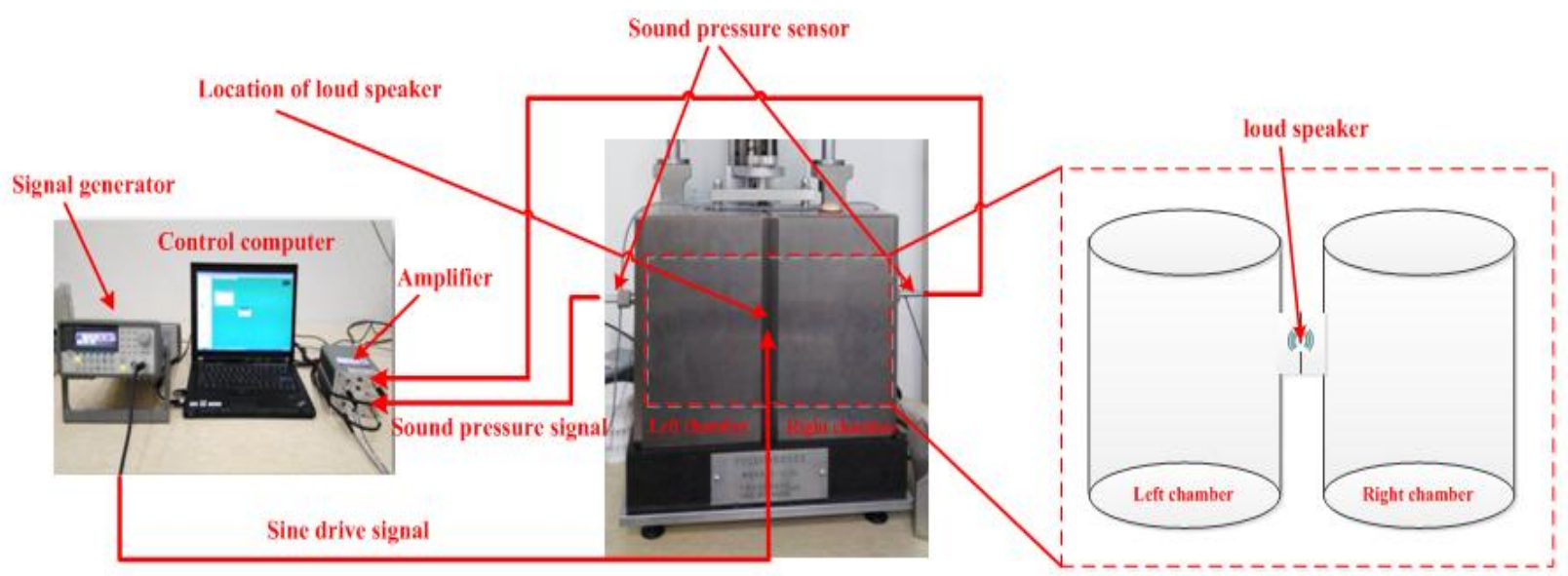

Figure 1. Schematic of the principle of the sound pressure generating in the measuring system

The loud speaker is driven by a single sinusoidal frequency signal generated by the signal generator. When the reflected sound waves generated by the vibration of the chamber and the loud speaker are not taken into account, the sound pressure in the chamber is generated by the sound pressure at the single frequency. Therefore, the actual measuring system is the interaction of sound and vibration, whose acoustic wave equation is

$$
\left[\begin{array}{cc}
M_{e}^{s} & 0 \\
M_{e}^{a s} & M_{e}^{a}
\end{array}\right]\left\{\begin{array}{c}
\ddot{u_{e}} \\
\ddot{P_{e}}
\end{array}\right\}+\left[\begin{array}{cc}
C_{e}^{s} & 0 \\
0 & C_{e}^{a}
\end{array}\right]\left\{\begin{array}{c}
\dot{u}_{e} \\
\dot{P_{e}}
\end{array}\right\}+\left[\begin{array}{cc}
K_{e}^{s} & K_{e}^{s a} \\
0 & K_{e}^{a}
\end{array}\right]\left\{\begin{array}{l}
u_{e} \\
P_{e}
\end{array}\right\}=\left\{\begin{array}{l}
0 \\
0
\end{array}\right\}
$$

in which $M_{e}^{a s}$ and $K_{e}^{s a}$ are coupling terms.

The degree of coupling is related to media density and wall stiffness. The wall of the chamber is aluminum alloy; the sound wave propagation medium is air, whose densities are quite different. Also, the chamber wall is thick and its stiffness is good, the sound and vibration coupling in the measuring system is small. Therefore, in the calculation of the chamber sound pressure distribution of this paper, the coupling of the vibration is not taken into account, the discrete equation of acoustic fluctuation can be simplified as Equation 3:

$$
M_{e}^{a} \ddot{P}_{e}+K_{e}^{a} P_{e}+\rho_{0} R_{e}^{\mathrm{T}} \ddot{u}_{e}=0
$$

Sound field distribution meets the acoustic wave equation as showed in Equation 3. The medium of sound wave propagation is air, whose viscosity is very small, the influence of the sound field distribution in the short distance is very small, and can be regarded as the ideal fluid with no viscosity. Acoustic amplitude is small. The sound pressure is very small compared with the static air pressure. In this FEM simulation, it is assumed that the air in the medium is uniform when no sound is disturbing, and the static pressure and the density of the air in the chamber are not changed with time and position. 
The finite element model of the chamber is shown in Figure 2. Only one of the chambers is selected for modelling because the left and right chambers are substantially symmetrical.

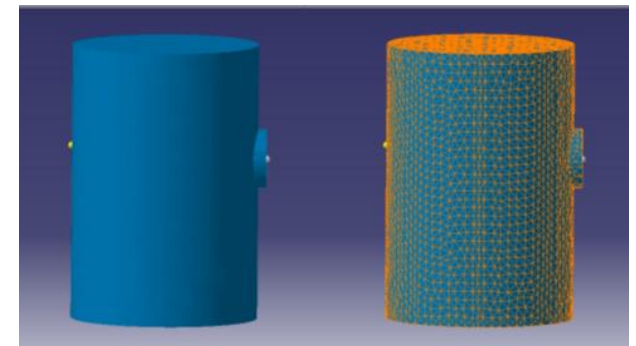

(a)

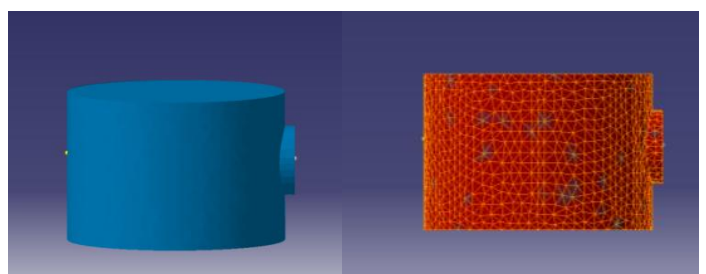

(b)

Figure 2. Modeling and meshing of finite element models for measuring large chambers (a) and small chambers (b).

One point sound source is put on the location of joint point of two chambers. The speaker's drive signal is the source of the sound pressure generated by the vibration of the speaker, whose characteristics, such as frequency and amplitude, have great influence on the stability and uniformity of the sound field in the chamber. The frequency of driving signal will affect the stability and precision of sound pressure ratio. The sound field distribution in the chamber finite element model is calculated by using the point acoustic source model as the excitation, and the distribution of sound pressure at each frequency is analyzed.

Two sets of finite element models with different geometrical dimensions are established to analyze the influence of air volume on sound pressure distribution and sound pressure ratio stability under the same frequency condition. The model uses a quadrilateral mesh for partitioning.

\section{Simulation Results Analysis and Discussion}

The experiments of acoustics measurement show that the most stable region of the sound pressure is mainly related to the frequency and amplitude of the sinusoidal driving signal. And experiments show that the higher measuring accuracy can be achieved at the lower frequency, especially around $43 \mathrm{~Hz}$. Thus to evaluate the efficient of the FEM model, the simulation analysis results selected low frequency $43 \mathrm{~Hz}$ and high frequency $2000 \mathrm{~Hz}$ for sound pressure contrast analysis, as is shown in Figure 3.

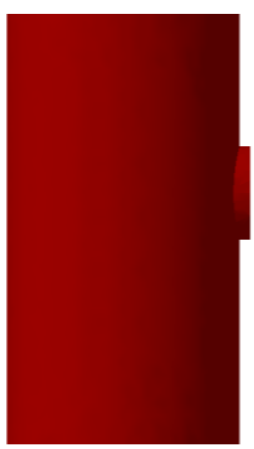

(a)

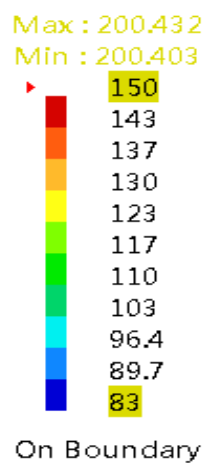

On Boundary

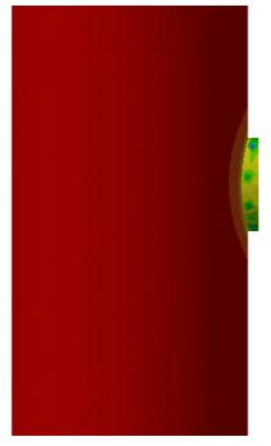

(b)

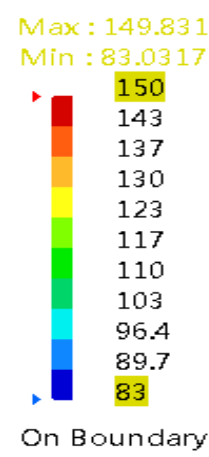

On Boundary

Figure 3. Contours of sound pressure level at $43 \mathrm{~Hz}$ (a) and $2000 \mathrm{~Hz}$ (b) calculated using a large chamber simulation model

Compared with high frequency, the distribution of sound pressure in the whole chamber is more uniform at low frequency, the range of sound pressure is only $0.029 \mathrm{~dB}$ (RMS) in chamber, and $66.799 \mathrm{~dB}$ (RMS) in high frequency. At high frequencies, the sound pressure level gradient is mainly distributed in the vicinity of the point sound source, in the far away from the point source, 
the sound pressure is relatively uniform. And the simulation results agree with the experimental results.

Figure 4 shows the sound field distributions in the large chamber and the small chamber at the drive frequency of $43 \mathrm{~Hz}$. As the simulation results show, the sound pressure distribution of the large chamber and the small chamber is relatively uniform at low frequency, the sound pressure level gradient is small, the distribution gradient of the small chamber sound pressure level is smaller, the maximum sound pressure level change is only 0.003 DB (RMS).

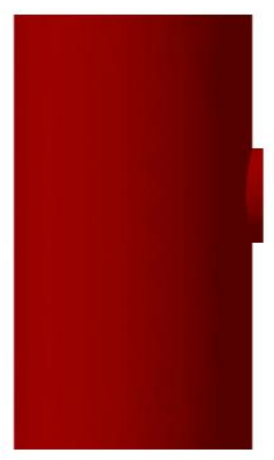

(a)

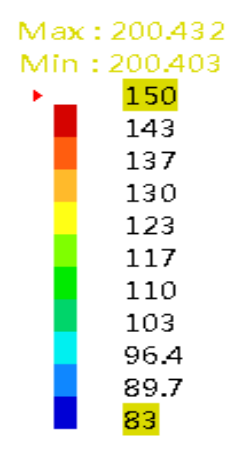

On Boundary

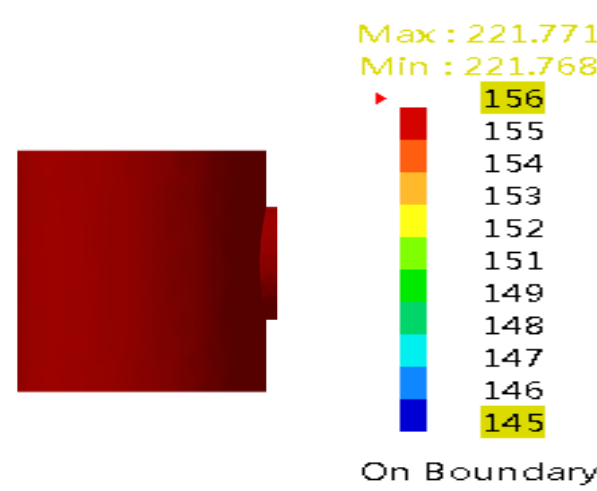

(b)

Figure 4. Contours of sound pressure level of the large chamber model (a) and the small chamber model (b) at $43 \mathrm{~Hz}$ Sine drive signal

Figure 5 shows the comparison of the sound pressure levels of the large chamber and the small chamber with drive frequencies from $1 \mathrm{~Hz}$ to $100 \mathrm{~Hz}$. Although when the drive frequency is large than $20 \mathrm{~Hz}$, the sound pressure in the same slope, the sound pressure level is higher about $9.8 \mathrm{~dB}$ (RMS) in small chamber than that in large chamber. This indicates that for small chamber, the sound pressure signals are more easily to be acquired for real measuring system.

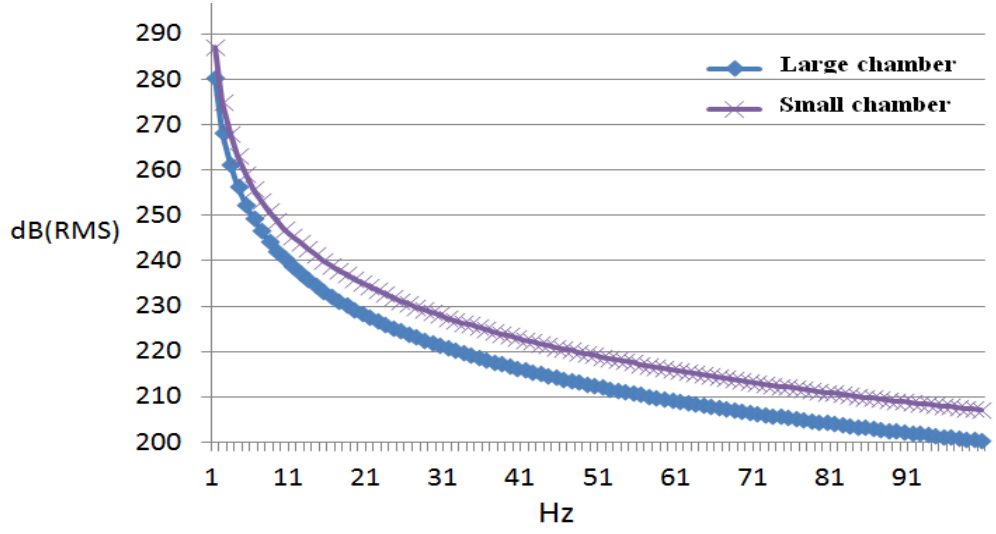

Figure 5. Comparison of the sound pressure levels of the large chamber and the small chamber with drive frequencies from $1 \mathrm{~Hz}$ to $100 \mathrm{~Hz}$

\section{Conclusions}

In this paper, A FEM model is designed to study the distribution and uniformity of sound pressure in volume determination with acoustic method. The FEM simulation results show that the sound pressure distribution gradient of the small chamber is better than large chamber. With the FEM model in this paper, the maximum sound pressure level change is only $0.003 \mathrm{DB}$ (RMS). And also for small chamber, the sound pressure signals are higher and more easily to be acquired for real measuring system. 


\section{Acknowledgements}

This research is supported by natural science foundation of china (NSFC) (Grant No. 51205379).

\section{References}

1. M. Ueki, T. Kobata, S. Mizushima, Y. Nezu, A. Ooiwa and Y. Ishii, Application of an acoustic volumeter to standard weights. 15th IMEKO World Congress. Osaka, 213-220 (1999).

2. T. Kobata, M. Ueki, A. Ooiwa and Y. Ishii, Mesurement of the volume of weights using an acoustic volumeter and the reliability of such measurement. Metrologia. 41, S75-S83 (2004).

3. M. Ueki, T. Kobata, K. Ueda and A. Ooiwa, Automated Volume Measurement for Weights using Acoustic Volumeter. 20th Conference on Measurement of Force. Mass and Torque. Mexico, (2007)

4. Weights of classes E1, E2, F1, F2, M1, M1-2, M2, M2-3, and M3. OIML regulation R111. edition $2004(\mathrm{E})$

5. M. Ueki, T. Kobata, K. Ueda and A. Ooiwa, Measurements of the volume of weights from $1 \mathrm{~g}$ to $50 \mathrm{~g}$ using an acoustic volumeter. SICE Annual Conference. Okayama. Japan, 1742-1747(2005).

6. I. Torigoc and Y. Ishii: Acoustic bridge volumeter. Trans.Soc.Instrum.Control Eng. E-1, 164170(2001).

7. M.H. Hu, J. Wang, Y. Zhang, C.Q. Cai and X. L. Wang: Experimental research on volume measurement method of weights based on acoustic principle. Chinese Journal of Scientific Instrument. 33, 2337-2342(2012). 